\title{
A base-calling algorithm for Tm-shifted melting curve SNP assay
}

\author{
Kung-Hao Liang ${ }^{1 *}$, Jun-Jeng Fen ${ }^{1,2}$, Hsien-Hsun Chang ${ }^{1,3}$, Hsei-Wei Wang ${ }^{2,4}$ and Yuchi Hwang ${ }^{1}$
}

\begin{abstract}
Background: Tm-shifted melting curve SNP assays are a class of homogeneous, low-cost genotyping assays. Alleles manifest themselves as signal peaks in the neighbourhood of theoretical allele-specific melting temperatures. Base calling for these assays has mostly relied on unsupervised algorithm or human visual inspection to date. However, a practical clinical test needs to handle one or few individual samples at a time. This could pose a challenge for unsupervised algorithms which usually require a large number of samples to define alleles-representing signal clusters on the fly.
\end{abstract}

Methods: We presented a supervised base-calling algorithm and software for Tm-shifted melting curve SNP assays. The algorithm comprises a peak detection procedure and an ordinal regression model. The peak detection procedure is required for building models as well as handling new samples. Ordinal regression is proposed because signal intensities of alleles $A A, A B$, and $B B$ usually follow an ordinal pattern with the heterozygous allele lie between two distinct homozygous alleles. Coefficients of the ordinal regression model are first trained and then used for base calling.

Results: A dataset of 12 SNPs of 44 unrelated persons was used for a demonstration purpose. The call rate is 99.6\%. Among the base calls, $99.1 \%$ are identical to those made by the sequencing method. A small fraction of the melting curve signals $(0.4 \%)$ is declared as "no call" for further human inspection. A software was implemented using the Java language, providing a graphical user interface for the visualization and handling of multiple melting curve signals.

Conclusions: Tm-shifted melting curve SNP assays, together with the proposed base calling algorithm and software, provide a practical solution for genetic tests on a clinical setting. The software is available in http://www. bioinformatics.org/mcsnp/wiki/Main/HomePage

\section{Background}

Discoveries of associations between genetic variants and clinical traits have improved our knowledge of human in health and disease [1]. Most of these findings came from research-phrase genome-wide association studies (GWAS) of various common-complex diseases [2-5]. Once validated in independent cohorts, these associations can facilitate the development of genetic tests for estimating personal disease risks. As GWAS gains popularity among clinical scientists, genetic tests are anticipated to play an increasingly important role in preventive and personalized healthcare systems.

\footnotetext{
* Correspondence: kunghao@gmail.com

${ }^{1}$ Vita Genomics Inc., Jungshing Road, Taipei County, 248 Taiwan

Full list of author information is available at the end of the article
}

Single nucleotide polymorphism (SNP) is an important class of human genomic variants widely assayed on GWAS. Current genetic tests are constructed on highdensity genome-wide assays [6] or low-cost, SNP-specific assays. The former aims to provide an extensive list of disease reports, while the latter gives results pertaining to a particular disease or a clinical trait.

A variety of assays has been developed for genotyping SNPs on the human DNA $[7,8]$. For research-phase projects, samples are usually collected in panels of many reaction wells and analyzed using unsupervised base calling algorithms. The entire panel is usually designated for a particular SNP. The fluorescent intensity signal of the entire panel is then clustered on-the-fly to make calls (e.g. [5] and the Rotor-Gene ScreenClust HRM Software). All three alleles of the SNP need to exist in

\section{Biomed Central}


the panel to define clusters properly. For cases when one allele type is rare, a larger pool of samples may be required to make the rare allele well represented [8]. In practice, many clinical labs received samples individually, each requiring the results to be delivered as soon as possible. Consequently, it is more practical and cheaper to run different assays (for different SNPs and/or different persons) concurrently in the same panel. Different SNPs may have different SNP-specific fluorescent distributions, prohibiting themselves to be clustered together. Therefore, a supervised base calling algorithm may be more adequate in a clinical setting. The SNP-specific coefficients are pre-trained to facilitate the base calling of individual samples.

The melting curve SNP genotyping assay, abbreviated as McSNP, is a class of simple, fast and relatively lowcost assays [9-19]. Among them, the Tm-shifted methods employ allele-specific primers which are designed to increase the melting temperature (Tm) difference between two allele-specific PCR duplex $[14,18,19]$. They are homogeneous assays where the entire process, including amplification and detection, is performed in solution within a single reaction well. Each allele manifested differently at its particular Tm. The base calling of Tm-shifted McSNP technology has relied mostly on unsupervised algorithm [18], user-specified cut-offs [16] or human visual inspection to date. Hence, we were motivated to propose a supervised base calling algorithm, enabling the McSNP assay a practical genetic test.

Denote the two alleles of a haploid SNP as A and B respectively. The goal of a base calling algorithm is to identify whether the assayed diploid SNP is homozygous AA (allele 1), heterozygous AB (allele 2), or homozygous BB (allele 3). Signals of AA, AB and BB usually follow a sequential order on a variety of assays including McSNP. Hence, we proposed an algorithm which comprises two procedures: (1) peak detection; and (2) base calling by an ordinal regression model. The peak detection procedure is required for both model training and the actual base calling. We also proposed the use SNPspecific offsets for adequate adjustments of the model to accommodate SNP-specific signal strengths. Samples of known alleles (determined by the conventional sequencing method) were used to train the coefficients of the algorithm, including the SNP-specific offsets and the ordinal regression coefficients. The trained model can then used for handling new coming samples.

\section{Methods}

\section{The Tm-shifted McSNP assay}

There are several variants of Tm-shifted McSNP assay $[14,18,19]$. We followed the protocol in [14] for primer design and experiment setting as an example. This technique requires two forward primers and one common reverse primer. The three primers form two primer pairs, amplifying allele-specific PCR products containing alleles A and B respectively. Reagents comprised SYBR Green PCR Master Mix (Applied Biosystem \#4309155) $(6 \mu \mathrm{L})$, two forward and one reverse SNP-specific primers $(0.4 \mu \mathrm{M}$ each), and the human genomic DNA (20 ng). The total reaction volume was $10 \mu \mathrm{L}$.

The assay started with a PCR procedure for DNA amplification. This started form the pre-incubation at $95^{\circ} \mathrm{C}$ to activate the Taq DNA polymerase $(10 \mathrm{mins})$, followed by 50 cycles of thermal cycling comprising (1) denaturation at $95^{\circ} \mathrm{C}(15 \mathrm{~s})$ and (2) primer annealing and extension at $60^{\circ} \mathrm{C}(1 \mathrm{~min})$. Afterwards, we continued the dissociation of the DNA duplex by gradually increasing the temperature up to $95^{\circ} \mathrm{C}$ at a temperature gradient of $0.2^{\circ} \mathrm{C} / \mathrm{min}$.

The Applied Biosystems ABI 7900HT instrument was used. The fluorescent signal was captured by the accompanied SDS 2.2 software. The theoretical temperature Tm was calculated using the dnaMate server [20] where a consensus melting temperature was calculated using the nearest-neighbour model based on three independent thermodynamic tables.

\section{Signal processing and peak Detection}

A disassociation curve, denoted as $F(T)$, is the fluorescent intensity plot captured during a dissociation process with increasing temperature $T$. Define a melting curve $M$ as the negative first-derivative of the disassociation curves $F[13]$, therefore

$$
M=-d F / d T
$$

Denote $\operatorname{Tm}(A)$ and $\operatorname{Tm}(B)$ as the theoretical melting temperatures of the PCR products, where $\operatorname{Tm}(A)<\operatorname{Tm}$ (B). Alleles manifest themselves as peaks on $M$ occurring near $\operatorname{Tm}(A)$ and $\operatorname{Tm}(B)$. Figure 1 illustrates the typical melting curve signals of the three types of alleles. A single peak on $M$ indicates a homozygous allele (Figure 1a and 1c), while two peaks indicate a heterozygous allele (Figure 1b). An optional Gaussian smoothing is applied to $M$ to suppress the small noisy fluctuations of the signal while preserving the major bending curves on $M$.

The proximity of $\operatorname{Tm}(A)$ and $\operatorname{Tm}(B)$, denoted as $R(A)$ and $R(B)$ respectively, are the main target regions of peak searching. This allows some degree of variation of the real Tm from the theoretical Tm.

$$
\begin{aligned}
& R_{A}=\left(2{ }^{*} \operatorname{Tm}(A) \times \operatorname{Tm}(B),(\operatorname{Tm}(A)+\operatorname{Tm}(B)) / 2\right] \\
& R_{B}=((\operatorname{Tm}(A)+\operatorname{Tm}(B)) / 2,2 * \operatorname{Tm}(B) \times \operatorname{Tm}(A)]
\end{aligned}
$$




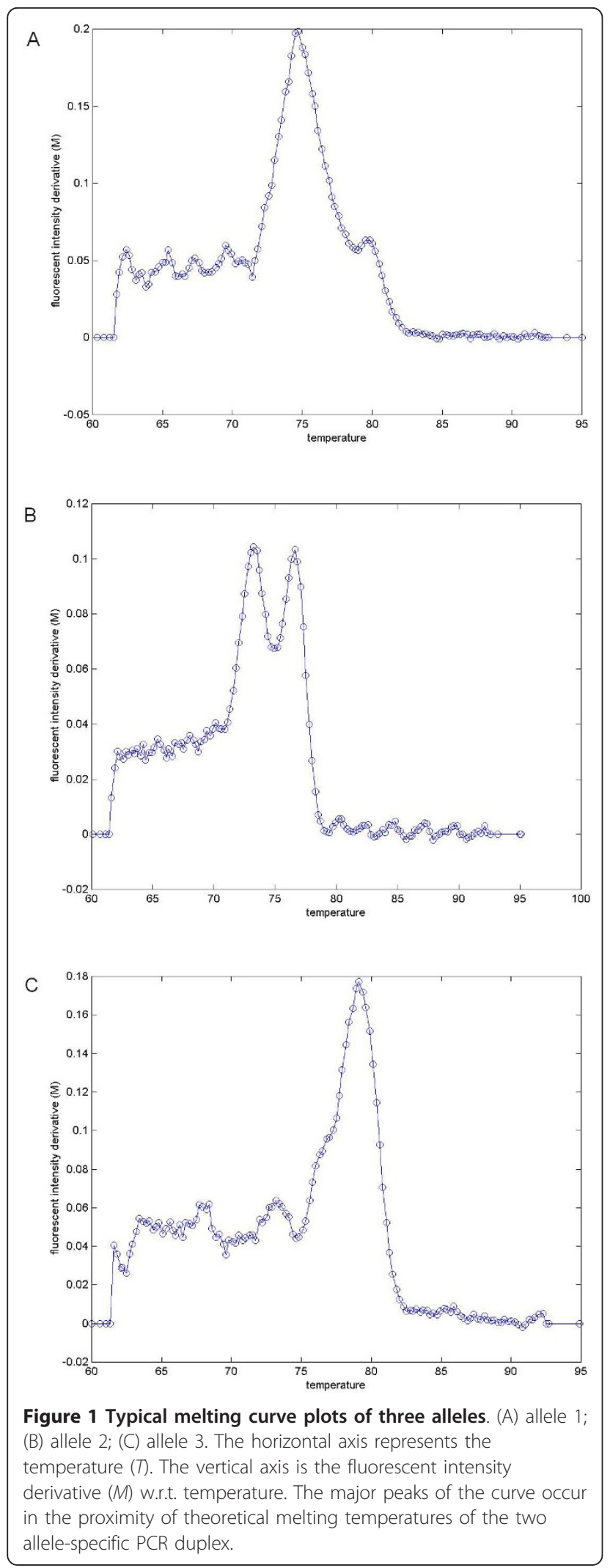

A derivative of the melting curve is then calculated within $R_{A}$ and $R_{B}$. A zero-crossing of the derivative either represents a peak (convex) or a valley (concave) on the melting curve. The peaks and valleys of a region are compared based on their height to find the tallest peak. The signal strengths of $A$ and $B$ alleles, denoted as $D_{A}$ and $D_{B}$ respectively, are the heights of the tallest peaks on $R_{A}$ and $R_{B}$, deducting the average height of the entire curve for normalization purposes. $D_{A}$ or $D_{B}$ takes the value of zero if no peak is detected in the corresponding region. If both $D_{A}$ and $D_{B}$ are 0 , then a "no call" is reported. Otherwise, a variable $x$ is introduced as the ratio of signal strengths:

$$
x=D_{B} /\left(D_{A}+D_{B}\right)
$$

\section{The ordinal regression model for base calling}

The base calling model was built upon the ordinal regression method, taking advantage of the fact that signal patterns of $\mathrm{AA}, \mathrm{AB}$ and $\mathrm{BB}$ usually follow a sequential order, with the heterozygous allele lie between two distinct homozygous alleles. Alleles 1 (AA), 2 (AB) and 3 (BB) constitute the three ordered categories of the response variable $Z$ of the regression model. Our implementation has three model coefficients $\alpha 1, \alpha 2$ and $\beta$. Given the coefficients, the cumulative response probabilities when $Z=\{$ allele 1$\}$ (denoted as $P(Z=\{1\})$ ) and $Z=$ \{alleles 1,2$\}$ (denoted as $P(Z=\{1,2\}))$, can be estimated using the following equations.

$$
\begin{aligned}
& \operatorname{logit}(P(Z=\{1\}))=\alpha 1-\beta X \\
& \operatorname{logit}(P(Z=\{1,2\}))=\alpha 2-\beta X
\end{aligned}
$$

The individual allele probability functions of alleles 2 and 3 can then be calculated by

$$
\begin{gathered}
P(Z=\{2\})=P(Z=\{1,2\})-P(Z=\{1\}) \\
P(Z=\{3\})=1-P(1,2)
\end{gathered}
$$

A probability margin $\rho$ was introduced. Bases are called by the following rules:

$$
\begin{aligned}
& \text { If }((\mathrm{P}(Z=\{2\})-\mathrm{P}(Z=\{1\}))>\rho \&(\mathrm{P}(Z=\{2\})-\mathrm{P}(Z=\{3\}))>\rho) \\
& \quad \text { "Allele 2"; } \\
& \text { else if }((\mathrm{P}(Z=\{3\})-\mathrm{P}(Z=\{1\}))>\rho \&(\mathrm{P}(Z=\{3\})-\mathrm{P}(Z=\{2\}))>\rho) \\
& \quad \text { "Allele 3"; } \\
& \text { else if }((\mathrm{P}(Z=\{1\})-\mathrm{P}(Z=\{2\}))>\rho \&(\mathrm{P}(Z=\{1\})-\mathrm{P}(Z=\{3\}))>\rho)
\end{aligned}
$$

"Allele 1";

else "no call"

If the difference the top two probabilities is smaller than $\rho$, then the base is called "no call" so as to trigger a warming message for human inspection. 
Table 1 List of SNPs

\begin{tabular}{cccc}
\hline ID & Gene Symbol & SNP & Allele (A/B) \\
\hline SNP1 & & rs2241796 & T/C \\
SNP2 & TGFBRAP1 & rs 1866040 & $\mathrm{G} / \mathrm{A}$ \\
SNP3 & & $\mathrm{rs} 2576737$ & $\mathrm{~A} / \mathrm{G}$ \\
SNP4 & & $\mathrm{rs} 518604$ & $\mathrm{C} / \mathrm{T}$ \\
SNP5 & CASP5 & $\mathrm{rs} 2282658$ & $\mathrm{C} / \mathrm{G}$ \\
SNP6 & & $\mathrm{rs} 484345$ & $\mathrm{~A} / \mathrm{G}$ \\
SNP7 & & $\mathrm{rs} 1699087$ & $\mathrm{G} / \mathrm{T}$ \\
SNP8 & ADAR & $\mathrm{rs} 903323$ & $\mathrm{~T} / \mathrm{C}$ \\
SNP9 & IFI44 & $\mathrm{rs} 2070123$ & $\mathrm{~T} / \mathrm{C}$ \\
SNP10 & & $\mathrm{rs} 305067$ & $\mathrm{G} / \mathrm{C}$ \\
SNP11 & ICSBP1 & $\mathrm{rs} 305088$ & $\mathrm{~A} / \mathrm{G}$ \\
SNP12 & & $\mathrm{rs} 870614$ & $\mathrm{G} / \mathrm{A}$ \\
\hline
\end{tabular}

These SNPs were assayed by both the sequencing and the McSNP methods for the demonstration of proposed algorithm.

\section{Results and Discussion}

\section{Determining coefficients}

The algorithm was trained on 44 human samples for a demonstration of this algorithm. Samples were from healthy Asian volunteers who has sign the inform consent form. Each sample was genotyped on a set of 12 SNPs (Table 1), producing 528 melting curve plots in total. The signal strength ratio $x$ was calculated for each plot (see Methods). These samples were also genotyped by the conventional sequencing method, serving as the expected calling results.

We aimed to obtain general coefficients rather than SNP specific coefficients to suit multiple SNPs. However, variations of $x$ do occur between different SNPs. Figure 2 shows the averages of $x$ for each allele of the 12 SNPs. To accommodate the variations of $x$, a SNPspecific offset $\delta$ is introduced which is calculated as follows. First, we take grand means $\langle x\rangle$ of the SNP-specific averages across all the 12 SNPs for alleles 1, 2

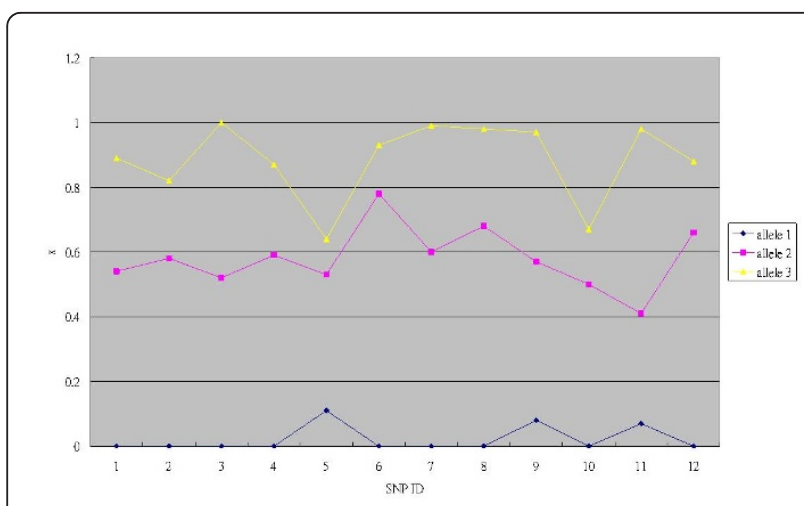

Figure 2 Allele-specific signal strength ratio $(x)$ derived from melting curves. Average $x$ of alleles 1,2 and 3 for each of the 12 SNPS.

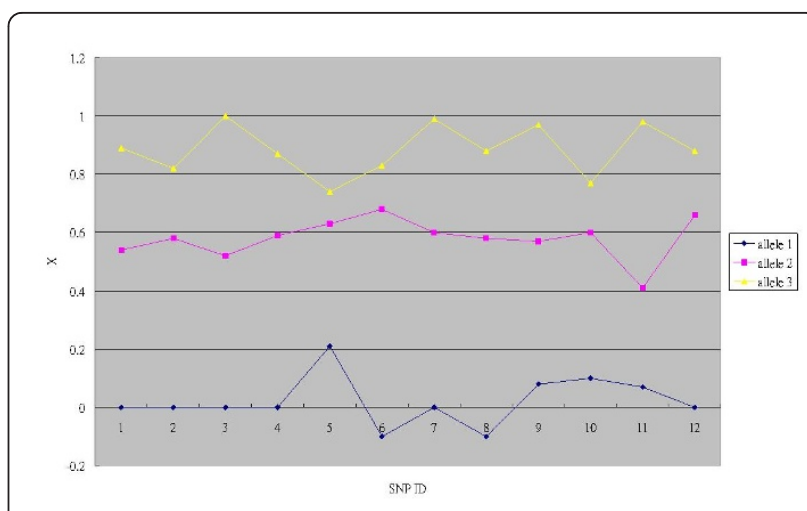

Figure 3 Adjusted signal strength ratio $(X)$. Average $X$ of alleles 1,2 and 3 for each of the 12 SNPs. SNPs 5, 6, 8 and 10 are offset from $x$ in Figure 2.

and 3. Second, $\delta$ 's are calculated by the SNP-specific averages of $x$ minus the grand means $\langle x\rangle$. We hoped to maintain zero offsets for most SNPs, therefore, the offsets were purposely kept in low resolution. They were rounding off to one decimal digit. As a consequence, 8 SNPs have zero offsets; SNPs 6 and 8 have an offset of 0.1 . SNPs 5 and 10 have an offset of -0.1 .

We further introduced the adjusted signal strength ratio $X$, defined as $X=x-\delta$. Compared with $x$, the distributions of $X$ of the 12 SNPs resemble each other better (Figure 3). Hence, $X$ is used for building the ordinal regression model. Based on all the 528 plots, $\alpha 1=15.3, \alpha 2=35.8, b=51$. The resulting allele probability functions $P(Z=\{1\}), P(Z=\{2\})$ and $P(Z=\{3\})$ are shown in Figure 4 which is the basis for subsequent base calling.

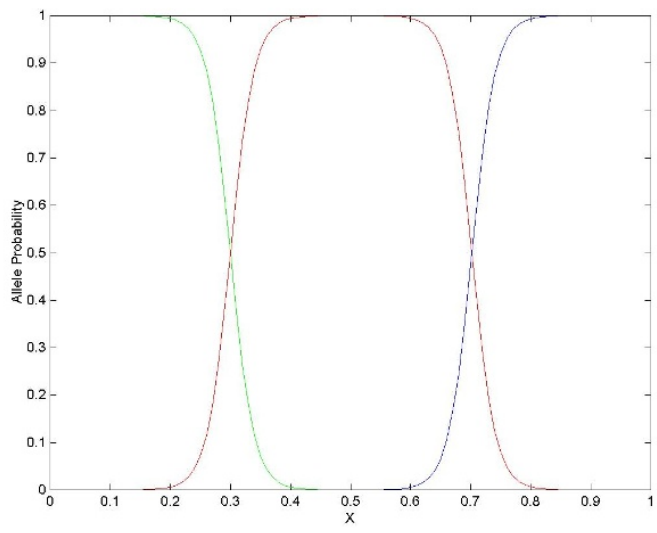

Figure 4 Allele probability functions. Allele probability, a function of $X$, is given by the ordinal regression model. Green: allele 1. Red: allele 2. Blue: allele 3. 
Table 2 SNP-specific calling performance

\begin{tabular}{ccccccccccccc}
\hline & SNP 1 & SNP 2 & SNP 3 & SNP 4 & SNP 5 & SNP 6 & SNP 7 & SNP 8 & SNP 9 & SNP 10 & SNP 11 & SNP 12 \\
\hline No calls & 0 & 0 & 0 & 0 & 0 & 1 & 1 & 0 & 0 & 0 & 0 \\
\hline \# discordant calls & 0 & 0 & 0 & 0 & 1 & 4 & 0 & 0 & 0 & 0 & 0 \\
\hline Concordance rate (\%) & 100 & 100 & 100 & 100 & 97.7 & 90.7 & 100 & 100 & 100 & 100 & 100 & 100 \\
\hline
\end{tabular}

The number of no calls, discordant calls and the concordance rates between the proposed algorithm and the sequencing method

Table 3 Comparison of the discordant calls between McSNP and sequencing

\begin{tabular}{lcccccc}
\hline & McSNP & Sequencing & $\boldsymbol{X}$ & $\begin{array}{c}\text { P(allele } \\
\text { 1) }\end{array}$ & $\begin{array}{c}\text { P(allele } \\
\text { 2) }\end{array}$ & $\begin{array}{c}\text { P(allele } \\
\text { 3) }\end{array}$ \\
\hline SNP5 & $\begin{array}{c}\text { allele 3 } \\
\text { (CC) }\end{array}$ & allele 2 (CG) & 0.73 & 0 & 0.19 & 0.81 \\
SNP6 & $\begin{array}{c}\text { allele 3 } \\
\text { (AA) }\end{array}$ & allele 2 (AG) & 0.71 & 0 & 0.40 & 0.60 \\
SNP6 & $\begin{array}{c}\text { allele 3 } \\
\text { (AA) }\end{array}$ & allele 2 (AG) & 0.72 & 0 & 0.28 & 0.72 \\
SNP6 & $\begin{array}{c}\text { allele 3 } \\
\text { (AA) }\end{array}$ & allele 2 (AG) & 0.71 & 0 & 0.40 & 0.60 \\
SNP6 & $\begin{array}{c}\text { No call } \\
\text { allele 3 } \\
\text { SNP6 }\end{array}$ & allele 2 (AG) & 0.70 & 0 & 0.52 & 0.48 \\
SNP7 & No call & allele 2 (GT) & 0.70 & 0 & 0.52 & 0.48 \\
\hline
\end{tabular}

Base calls, allele signals $(\mathrm{X})$ and their corresponding allele probabilities are presented.
$\mathrm{X}$ and $\mathrm{x}$ is only different by an offset $\delta$ which takes one of three values, $-0.1,0$ and 0.1 . Referring to the ordinal regression equation:

$$
\begin{aligned}
\operatorname{logit}(P(Z=\{1\})) & =\alpha 1-\beta X \\
& =\alpha 1-\beta(x-\delta) \\
& =(\alpha 1-\delta)-\beta x,
\end{aligned}
$$

the three offsets effectively generates three different models to accommodate the variation of signal strength ratios of the 12 SNPs. The model with zero offsets may have the widest use because it is built upon a large portion of the training dataset.

\section{Base calling performance}

The margin of probability $\rho$ was set at 0.05 for the base calling. The performance was summarized in Table 2 .
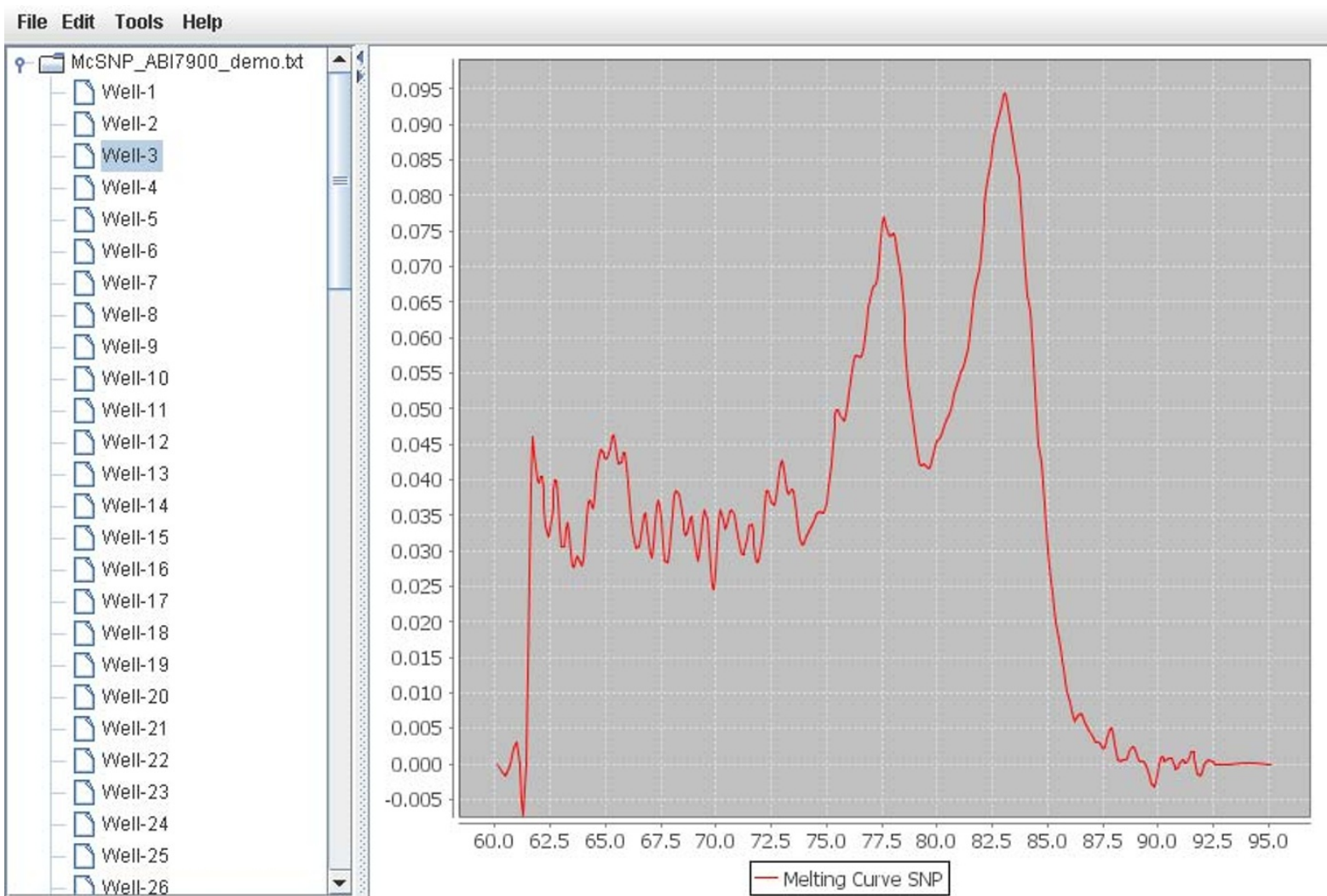

Figure 5 The graphical user interface of the software. The software was implemented in Java for providing a convenient interface for data visualization and handling. 
The call rate is $99.6 \%$ because two SNPs are declared as no calls. Among the 12 SNPs, 10 SNPs reached 100\% concordance rate, defined as the percentage of base calls identical to those from the sequencing method. The average concordance rate is $99.1 \%$. For all the discordant callings, base calls by the sequencing method were allele 2 , while by McSNP were allele 3 (Table 3 ). This is because the melting-curve signals on the first allele is relatively weak, occasionally missing, thus the first alleles are not easily detected by the base calling algorithm.

\section{The software}

A software was developed on the Java programming language to implement the proposed algorithm and also provide a user friendly graphical interface. The software can handle a fluorescent signal exports from SDS2.2 and then calculate the signal strength ratio $x$. Given SNPspecific offsets, theoretical melting temperatures and the coefficients of the ordinal regression model, the software can then make calls. The graphical user interface was designed for the ease of signal visualization and manipulation (Figure 5). The software is available in http:// www.bioinformatics.org/mcsnp/wiki/Main/HomePage.

\section{Conclusions}

The supervised base calling algorithm and software were designed for the clinical use of Tm-shifted melting curve SNP genotyping assays. A supervised algorithm was designed due to practical considerations of its clinical use. An ordinal regression model was employed to capture the sequential order of average allele signals. A set of general coefficients were provided based on a demonstration dataset. Clinicians can conduct the base calling using the general coefficients, or carry out the coefficients training and the subsequent base calling themselves

Although this algorithm was developed upon the Tmshifted McSNP data, it can be adapted for other McSNP methods. Particularly, this line of technology is still evolving and new improvements of the analytical chemistry appear gradually. The proposed algorithm and training strategy can also evolve accordingly. By the combination of efficient base calling software and a small-scale human inspection, a practical SNP tests can be established.

\footnotetext{
Author details

${ }^{1}$ Vita Genomics Inc., Jungshing Road, Taipei County, 248 Taiwan. ${ }^{2}$ Institute of Biomedical Informatics, National Yang-Ming University, Linong Street, Taipei, 112 Taiwan. ${ }^{3}$ Graduate Institute of Biomedical Materials and Engineering, Taipei Medical University, Wu-Hsing Street, Taipei, 110 Taiwan. ${ }^{4}$ Institute of Microbiology and Immunology, National Yang-Ming University, Li-Nong Street, Taipei, 112 Taiwan.
}

\section{Authors' contributions}

$\mathrm{KHL}$ designed the algorithm, implemented the prototype of the core algorithm and drafted the manuscript. JJF implemented the JAVA version of the software with friendly graphical user interface. $\mathrm{HHC}$ conducted the MCSNP and sequencing experiments. HWW contributed on the study design and data analysis. YCH conceived and coordinated the study. All authors read and approved the final manuscript.

\section{Competing interests}

The authors declare that they have no competing interests.

Received: 14 July 2010 Accepted: 20 January 2011

Published: 20 January 2011

\section{References}

1. Hirschhorn JN: Genomewide association studies: Illuminating biologic pathways. N Engl J Med 2009, 360(17):1699-1701.

2. Sladek R: A genome-wide association study identifies novel risk loci for type 2 diabetes. Nature 2007, 445:881-885.

3. SLEGEN: Genome-wide association scan in women with systemic lupus erythematosus identifies susceptibility variants in ITGAM, PXK, KIAA1542 and other loci. Nat Genet 2008, 40(2):204-210.

4. Thomas G: Multiple loci identified in a genome-wide association study of prostate cancer. Nat Genet 2008, 40:310-315.

5. Wellcome Trust Case Control Consortium: Genome-wide association study of 14000 cases of seven common diseases and 3000 shared controls. Nature 2007, 447:661-678.

6. Fan JB, Chee MS, Gunderson KL: Highly parallel genomic assays. Nat Rev Genet 2006, 7:632-644.

7. Shi MM: Enabling large-scale pharmacogenetic studies by highthroughput mutation detection and genotyping technologies. Clin Chem 2001, 47:164-172.

8. Callegaro A, Spinelli R, B L, Bicciato S, Caristina L, Censuales S, De Bellis G, Battaglia C: Algorithm for automatic genotype calling of single nucleotide polymorphisms using the full course of TaqMan real-time data. Nucleic Acids Res 2006, 34:e56.

9. Akey JM, Sosnoski D, Parra E, Dios S, Heister K, Su B, Bonilla C, Jin L, Shriver MD: Melting curve analysis of SNPs (McSNP): a gel free and inexpensive approach for SNP genotyping. BioTechniques 2001, 30:358-367.

10. Liew M, Pryor R, Palais R, Meadows C, Erall M, Lyon E, Wittwer C: Genotyping of single-nucleotide polymorphisms by high-resolution melting of small amplicons. Clin Chem 2004, 50(7):1156-1164.

11. Liew M, Nelson L, Margraf R, Mitchell S, Erall M, Mao R, Lyon E, Wittwer C: Genotyping of human platelet antigens 1 to 6 and 15 by high resolution amplicon melting and conventional hybridization probes. J Mol Diag 2006, 8(1):97-103.

12. Lipsky RH, Mazzanti CM, Rudolph JG, Xu K, Vyas G, Bozak D: DNA melting analysis for detection of single nucleotide polymorphisms. Clin Chem 2001, 47:635-644.

13. Herrmann MG, Durtschi JD, Bromley LK, Wittwer CT, Voelkerding KV: Amplicon DNA melting analysis for Mutation Scanning and Genotyping: CrossPlatform Comparison of Instruments and Dyes. Clin Chem 2006, 52:494-503.

14. Papp AC, Pinsonneault JK, Cooke G, Sadee W: Single nucleotide polymorphism genotyping using allele-specific PCR and fluorescence melting curves. BioTechniques 2003, 34(5):1067-1072.

15. Ririe KM, Rasmussen RP, Wittwer $C T$ : Product differentiation by analysis of DNA melting curves during the polymerase chain reaction. Anal Biochem 1997, 245:154-160

16. Ye J, Parra EJ, Sosnoski DM, Hiester K, Underhill PA, Shriver MD: Melting curve SNP (McSNP) genotyping: a useful approach for diallelic genotyping in forensic science. J Forensic Sci 2002, 47(3):593-600.

17. Zhou L, Wang L, Palais R, Pryor R, Wittwer CT: High-resolution DNA melting analysis for simultaneous mutation scanning and genotyping in solution. Clin Chem 2005, 51(10):1770-1777.

18. Wang J, Chuang K, Ahluwalia M, Patel S, Umblas N, Mirel D, Highchi R, Germer S: High-throughput SNP genotyping by single-tube PCR with Tm-shift primers. BioTechniques 2005, 39(6):885-893.

19. Germer S, Higuchi R: Single-tube genotyping without oligonucleotide probes. Genome Res 1999, 9:72-78.

20. Panjkvoich A, Norambuena T, Melo F: dnaMATE: a consensus melting temperature prediction server for short DNA sequences. Nucleic Acids Res 2005, 33:W570-W572.

doi:10.1186/2043-9113-1-3

Cite this article as: Liang et al:: A base-calling algorithm for Tm-shifted melting curve SNP assay. Journal of Clinical Bioinformatics 2011 1:3. 